Cambouis, la revue des sciences sociales aux mains sales

\title{
Équipements collectifs et ségrégation sociale
}

\section{Chronique méthodologique d'une recherche}

\author{
Monique Pinçon-Charlot \\ Centre de Sociologie Urbaine \\ Edmond Préteceille \\ Centre de Sociologie Urbaine \\ Paul Rendu \\ Centre de Sociologie Urbaine
}

Résumé: Dans le cours de nos recherches sur les politiques urbaines, l'analyse statistique de la distribution spatiale et sociale des équipements collectifs à l'échelle d'une région urbaine socialement diversifiée nous a paru une étape indispensable pour l'étude des effets sociaux de ces politiques. Rassembler, traiter, interpréter la somme, considérable, des informations nécessaires sur un ensemble social aussi vaste que la région parisienne demande un travail tel qu'il implique, en fait, la coopération de plusieurs chercheurs pendant plusieurs années. Du point de vue des rapports entre les chercheurs eux-mêmes, ce n'est pas toujours simple, dans la mesure où il faut gérer en permanence la contradiction entre la nécessité d'une problématique commune définissant précisément le programme de travail et les différences inévitables (et productives à condition qu'elles ne soient pas paralysantes) de sensibilité théorique, de manière de travailler, d'écrire... De plus, l'idéologie professionnelle dominante dans la recherche en sciences sociales est fortement individualiste, et les instances scientifiques, commissions du CNRS entre autres, ont une attitude encore trop réservée par rapport aux travaux collectifs. La réalisation de travaux empiriques d'une certaine ampleur exige également des moyens importants. Ces travaux présentés ici ont pu être menés à leur terme grâce principalement aux financements du Commissariat Général au Plan puis à ceux du Plan-Construction. Bien sûr, ce n'est pas très élégant de parler d'argent dans une discussion épistémologique. Et puis nous avions « choisi » les plus mauvaises conditions possibles pour faire ce travail : sur contrats (il nous en a fallu sept ou huit pour l'ensemble du programme, gymnastique passible de la réprobation de la Cour des comptes, faut-il l'avouer ?) devant financer nos salaires, les charges sociales, etc., avant même les frais de recherche, et tout cela en pleine période de récession de la recherche contractuelle et de désintérêt pour les travaux sociologiques quantitatifs de la part de nombreuses administrations. Et il faut bien dire que le long travail de « digestion théorique » des résultats, indispensables pour donner tout son sens au travail statistique, n'a pu avoir lieu vraiment que grâce à notre intégration au CNRS en cours de route.

Date de publication : 8/7/2021

Dossier : Sociologie et statistique

Comment citer : $10.52983 /$ crev.vi0.51 
Licence:Cambouis publie ses contenus selon les termes de la Licence Creative Commons Attribution - Pas d'Utilisation Commerciale - Pas de Modification 4.0 International. Les auteurices gardent leurs droits de propriété intellectuelle pleine et entière sur leurs articles. 


\title{
Équipements collectifs et ségrégation sociale
}

\section{Chronique méthodologique d'une recherche}

\author{
Monique Pinçon-Charlot \\ Centre de Sociologie Urbaine \\ Edmond Préteceille \\ Centre de Sociologie Urbaine \\ Paul Rendu \\ Centre de Sociologie Urbaine
}

Dans le cours de nos recherches sur les politiques urbaines, l'analyse statistique de la distribution spatiale et sociale des équipements collectifs à l'échelle d'une région urbaine socialement diversifiée nous a paru une étape indispensable pour l'étude des effets sociaux de ces politiques.

Rassembler, traiter, interpréter la somme, considérable, des informations nécessaires sur un ensemble social aussi vaste que la région parisienne demande un travail tel qu'il implique, en fait, la coopération de plusieurs chercheurs pendant plusieurs années.

Du point de vue des rapports entre les chercheurs eux-mêmes, ce n'est pas toujours simple, dans la mesure où il faut gérer en permanence la contradiction entre la nécessité d'une problématique commune définissant précisément le programme de travail et les différences inévitables (et productives à condition qu'elles ne soient pas paralysantes) de sensibilité théorique, de manière de travailler, d'écrire... De plus, l'idéologie professionnelle dominante dans la recherche en sciences sociales est fortement individualiste, et les instances scientifiques, commissions du CNRS entre autres, ont une attitude encore trop réservée par rapport aux travaux collectifs. On accepte plus facilement la division universitaire-mandarinale du travail, qui est une division hiérarchique entre travail théorique et travail empirique, aux multiples conséquences nocives dont les moindres ne sont pas théoriques, en dépit de camouflages souvent habiles.

La réalisation de travaux empiriques d'une certaine ampleur exige également des moyens importants. Ces travaux présentés ici ont pu être menés à leur terme grâce principalement aux financements du Commissariat Général au Plan puis à ceux du Plan-Construction. Bien sûr, ce n'est pas très élégant de parler d'argent dans une discussion épistémologique. Et puis nous avions « choisi » les plus mauvaises conditions possibles pour faire ce travail : sur contrats (il nous en a fallu sept ou huit pour l'ensemble du programme, gymnastique passible de la réprobation de la Cour des comptes, faut-il l'avouer ?) devant financer nos salaires, les charges sociales, etc., avant même les frais de recherche, et tout cela en pleine période de récession de la recherche contractuelle et de désintérêt pour les travaux sociologiques quantitatifs de la part de nombreuses administrations. Et il faut bien dire que le long travail de « digestion théorique » des résultats, indispensables pour donner tout son sens au travail statistique, n’a pu avoir lieu vraiment que grâce à notre intégration au CNRS en cours de route. 
Ces difficultés cumulées ont tendu à laisser aux administrations gestionnaires le quasi-monopole de production de données statistiques dans bien des domaines, à côté des champs couverts par des organismes spécialisés comme l'Insee ou le Credoc. C'est en partie le résultat d'une certaine politique de la recherche contractuelle de rendre difficiles des programmes empiriques ambitieux et de faire jouer au sociologue le rôle, stérile à force d'être trop exclusif, d'agitateur d'idées, de (mauvaise) conscience critique, de mouche du coche, de producteur d'un discours d'autant plus facilement disqualifiable ou récupérable qu'il est moins fondé empiriquement. C'est aussi le résultat de l'intériorisation de cette politique et de certaines tendances du milieu universitaire par les chercheurs eux-mêmes de détourner ceux-ci des occasions intéressantes qui passent à leur portée. Dans la lettre mensuelle du CNRS, une petite annonce offrait un poste au cœur de la production des statistiques de l'Éducation Nationale ; ce poste est resté longtemps non pourvu, jusqu'à ce jour peut-être.

Un autre obstacle à l'intégration théorique des méthodes quantitatives en sociologie tient à la séparation entre les chercheurs recueillant les données et interprétant les résultats et les spécialistes des méthodes censés leur fournir des outils neutres et universels. Tout ce qui a couleur mathématique inspire encore souvent au sociologue effroi et/ou respect absolu ; on n'ira donc pas voir ce que c'est dans le détail ni comment ça marche. On se défiera absolument de l'enfer des chiffres, ou on fera confiance aveuglément à la Science et à ses boîtes noires, en espérant quand même recueillir au passage un peu de la «poussière de fée » de la scientificité mathématique-statistique-informatique.

Réciproquement, le statisticien ou l'informaticien, s'il se hasarde hors de l'univers touffu mais logique et conceptuellement cohérent des théorèmes et algorithmes, se sent mal à l'aise sur le terrain, mouvant et traversé de failles et de contradictions, des notions, concepts, idéologies et théories multiples et concurrentes des sciences sociales et humaines.

Ce cloisonnement ne fait que conforter ce que nous appellerons l'opportunisme méthodologique. Nous avons, pour notre part, tenté de l'éviter en contrôlant théoriquement et directement les étapes successives de la démarche empirique, le choix et l'utilisation des méthodes de traitement, selon des modalités qui seront décrites plus loin.

$\mathrm{Au}$-delà de notre expérience particulière, il semble d'ailleurs que nous soyons aujourd'hui à un moment de restructuration théorico-empirique de la discipline. D'importants progrès théoriques ont été effectués dans divers domaines de la sociologie au cours des dernières années, en particulier en sociologie urbaine, et ces progrès théoriques produisent aujourd'hui, de par leur existence même et par leur développement propre, des exigences nouvelles de recherche empirique plus complexe, de plus grande ampleur, et surtout maîtrisée théoriquement, homogène à ces progrès, alors que les travaux empiriques effectués jusque-là ont relevé encore trop souvent du bricolage artisanal et illustrateur, de l'utilisation opportuniste de méthodes souvent inadaptées. Ainsi les progrès théoriques créent-ils des besoins méthodologiques nouveaux. De surcroît, le développement rapide de l'informatique donne des moyens potentiels de traitement de données nombreuses et complexes et ouvre des possibilités méthodologiques nouvelles. L'intérêt pour les méthodes d'analyse multidimensionnelle du type de l'analyse des correspondances en porte témoignage.
1 Ces questions ont été développées plus longuement dans Préteceille (1979a). 


\section{Les motivations théoriques d'une étude statistique}

Les recherches antérieures sur les effets sociaux des politiques urbaines avaient souligné l'importance des équipements collectifs et de la ségrégation sociale sans toutefois que leurs rapports aient été systématiquement étudiés. Les hypothèses plus ou moins divergentes sur la reproduction de la force de travail, sur la disciplinarisation, sur les concessions à la nouvelle petite bourgeoisie s'appuyaient au mieux sur des illustrations ponctuelles par quelques monographies urbaines difficilement généralisables. D'où le projet ${ }^{2}$ de les confronter à une étude statistique qui ne réduirait a priori ni la complexité de la structure urbaine, ni celle de la structure sociale, qui s'efforcerait aussi de tenir compte de l'unité interne relative des modes de vie, en étudiant simultanément l'ensemble des équipements accessibles dans l'environnement du lieu de résidence, au lieu d'un seul secteur, tel que la santé ou l'école. Les équipements spécialisés jouent en effet des rôles différents dans l'organisation des modes de vie et des pratiques, non pas seulement en raison des caractéristiques qui leur sont propres mais aussi en fonction des modalités selon lesquelles ils s'articulent les uns aux autres sur le plan local (espace où s'organisent les consommations).

\section{Données, trouvées, produites?}

Des hypothèses théoriques au départ de la recherche — hypothèses sur les dimensions pertinentes d'analyse des processus de socialisation de la consommation et sur l'organisation socio-spatiale de ces processus (Préteceille et al, 1975) — découlaient un certain nombre de principes pour la construction des données empiriques. Ces principes portaient, d'une part, sur la définition des différents aspects du phénomène équipement collectif qu'il était nécessaire de considérer, d'autre part, sur la nécessité de préciser les rapports entre les équipements et la population de leurs utilisateurs potentiels. Et cela d'une manière telle que l'on puisse ensuite pour chaque groupe social présent dans l'espace urbain reconstituer le système des équipements auxquels il a potentiellement accès. Et, enfin, ils impliquaient la construction de données qui permettent de couvrir entièrement et de façon homogène l'ensemble de l'espace urbain de la région parisienne.

La possibilité de réaliser un tel projet - concrètement, étudier l'ensemble des équipements accessibles ou présents dans chacune des 468 communes urbaines de la région Île-de-France et des 80 quartiers de Paris — dépend d'abord de la capacité à produire les informations nécessaires, qui doivent être théoriquement pertinentes et statistiquement synthétisables. Il s'est agi en fait de trouver et d'utiliser des informations produites et rassemblées dans d'autres buts. D'où des difficultés qui ne sont pas que techniques mais aussi théoriques : multiples décalages entre nos besoins et le savoir utile aux diverses administrations constructrices ou gestionnaires dont le regard spécifique sur la société colore le monopole de production d'informations statistiques ; multiples manques, réductions, homogénéisations, etc. que ne compensent pas nos sélections et transformations, avec lesquelles il faut pourtant passer un compromis en prenant garde que celui-ci soit provisoire, négocié et mémorisé.

Les quelque quatre cents indicateurs d'équipements que nous avons construits caractérisent une douzaine de « secteurs » d'équipements ${ }^{3}$. Sept d'entre eux ont été étudiés à partir de données fournies par les administrations d'État directement compétentes pour la réalisation et la gestion desdits équipements, données enregistrées dans le cours même de leur activité. Trois
2 Projet dont la problématique et la stratégie empirico-théorique sont présentées dans Préteceille $e t$ al (1975).

3 Les principes de construction des indicateurs, les sources utilisées et les valeurs, pour chaque unité urbaine, des indicateurs construits ont été publiés dans Chantrein $e t$ al (1976-1977). La phase de collecte des données puis celle de définition et de calcul des indicateurs ont été réalisées en collaboration avec l'Atelier Parisien d'Urbanisne (APUR).

.


à partir du résultat d'enquêtes menées pour l'administration par des services d'études publics et parapublics, à quoi on peut en ajouter deux autres pour lesquels les données provenaient essentiellement de l'Insee. Si l'on néglige les enquêtes complémentaires ou les sources annexes, dix sur douze des secteurs ont été traités avec des données produites en liaison étroite avec la pratique administrative et ses besoins en matière d'information.

L'information la plus facilement accessible (au chercheur, pas au public) est, le plus souvent, celle qui concerne les caractéristiques physiques (en liaison sans doute avec la gestion budgétaire des opérations d'investissement). Les données sur le personnel sont plus difficiles à obtenir, surtout si l'on s'intéresse aux effectifs réels, aux qualifications et aux statuts. Les données de fonctionnement (crédits, moyens matériels) sont également difficiles à obtenir. Enfin, et surtout, ce qui est le plus systématiquement absent, ce sont des données qui permettraient d'apprécier le fonctionnement interne réel des équipements dans ses variations possibles quelquefois fort larges autour des normes officielles (exemple : crèches ouvertes et éducatives ou crèches «médicalisées ») et dans la réalité des pratiques qui s’y déploient (dont la fréquentation par les différentes classes ou catégories sociales est un des éléments descriptifs importants). Certaines pouvaient être obtenues par enquête auprès des gestionnaires (tarifs, horaires, règlement...) mais d'autres informations impliquent en fait une observation directe des pratiques, donc une enquête sur place, et pouvaient d'ailleurs difficilement être transcrites en indicateurs chiffrés simples dès lors en particulier qu'on a affaire à des processus sociaux contradictoires.

Au bout du compte, sur près de quatre cents indicateurs calculés, les deux tiers portent sur des caractéristiques physiques (y compris accessibilité, variété et aspects qualitatifs divers des installations), le tiers seulement sur des caractéristiques ayant à voir directement ou indirectement avec le personnel et les moyens de fonctionnement. Signalons toutefois que la répartition de ces deux catégories est très inégale suivant les secteurs d'équipement.

Il est certain que la tendance à réduire les consommations collectives aux équipements comme objets matériels et opérations d'investissement a un effet objectif, structurel, de «naturalisation » de ces équipements. Au sens où est écartée la structure des rapports sociaux de consommation et toutes les questions, toutes les contradictions qui lui sont liées : problèmes de la ségrégation sociale interne, problèmes des effets idéologiques du fonctionnement (pas seulement en termes de discours), problèmes du pouvoir, des mécanismes de renforcement de l'hégémonie, revendications, luttes, pratiques différentes des personnels, des usagers... Il faut donc, pour interpréter correctement les résultats statistiques de cette recherche, bien tenir compte, pour chaque type d'équipement, des biais spécifiques des données de départ et les considérer comme des résultats partiels qui ne peuvent prendre tout leur sens sociologique qu'en rapport avec l'étude, par d'autres moyens, des aspects qui ont échappé à cette démarche ${ }^{4}$.

Soulignons enfin que la construction d'un nombre élevé d'indicateurs correspond à la volonté de saisir au mieux, compte tenu des données de départ, les aspects divers d'un même type d'équipement, les différentes dimensions selon lesquelles on peut analyser son rapport aux utilisateurs potentiels. Ce choix de principe est important pour ne pas réduire a priori cette diversité, qui était au contraire une dimension d'analyse soulignée par nos hypothèses de départ dans l'éventualité de différenciations sociales par type de structures d'équipement et pas seulement par un plus ou moins haut niveau d'équipement.
4 Sur cette question des logiques institutionnelles de production des statistiques, on trouvera un bon exemple d'analyse précise des « données » disponibles dans un secteur d'équipement dans Briand $e t$ al (1979). 


\section{Quel traitement?}

L'analyse théorique de la diversité des consommations collectives et de la multiplicité, a priori irréductible, de leurs aspects permet de comprendre notre intérêt pour les méthodes d'analyse multidimensionnelle, redoublé donc par les hypothèses sur le caractère également multidimensionnel de la structuration socio-spatiale des équipements collectifs.

Le choix de méthodes de statistique descriptive correspondait à notre refus de recourir à des méthodes du type modèle ou de tout genre impliquant des hypothèses probabilistes ou des schémas de causalité implicites ou explicites. Nous avons voulu nous en tenir, dans un premier temps, à décrire la structure, dégager les principes d'organisation du système des équipements collectifs.

Il reste que la description elle-même implique des opérations théoriques. Car, pour ne prendre que ce problème, décisif il est vrai, un nuage de points n'a pas de forme si l'on n'a pas défini une métrique. Et, dans une certaine mesure, c'est la métrique qui produit la forme. Quel est le sens sociologique du point de vue des rapports entre des données de nature hétérogène, de la définition d'une métrique commune, quelles sont les conséquences de ces limites sur les résultats eux-mêmes, et enfin y a-t-il des différences dans les réponses à ces questions pour des métriques différentes, euclidienne ou du chi $^{2}$ par exemple?

Nous n'avons pas, au départ, et malgré plusieurs discussions avec les spécialistes, trouvé de raisons théoriques justifiant du choix de l'une d'entre elles, et c'est finalement la métrique euclidienne qui a été retenue, surtout pour des raisons d'opportunité (possibilité d'utiliser le programme PROFIL d'analyse en composantes principales mis en ouvre à l'APUR, ce qui nous donnait simultanément l'accès à d'autres variables descriptives dont nous avions besoin). Par la suite, notre culture et notre réflexion méthodologiques s'approfondissant, nous avons eu quelques remords en faveur de l'analyse des correspondances dont il nous a semblé qu'elle était plus économe d'hypothèses implicites d'additivité, bien qu'elle n'y échappe pas totalement (Dreyfus, 1975). Par la suite, Jean-Pierre Fénelon nous a rassurés en indiquant que la distinction entre les deux métriques était, pour ce type d'utilisation, un problème surévalué. Les deux méthodes étant, sur le plan mathématique, des variantes de l'analyse canonique, équivalentes par transformation du recodage des données.

Nous avons tout d'abord traité nos indicateurs par secteurs d'équipements redéfinis en fonction du critère d'homogénéité des consommations collectives en question afin, d'une part, de minimiser les éventuels effets négatifs de l'application d'une métrique commune à des variables décrivant des objets sociaux trop différents, d'autre part, de bien contrôler l'interprétation des facteurs dégagés, par l'étude fine de leurs rapports aux indicateurs traités ${ }^{5}$.

À partir de ces analyses sectorielles, et compte tenu de la forte similitude des structures dégagées dans chacune d'entre elles, nous avons effectué un traitement intersectoriel sur 178 indicateurs. Ceux-ci ont été sélectionnés de façon à avoir une représentation complète de tous les secteurs tout en évitant que certains aient un poids plus important du seul fait de la plus grande richesse des sources d'information, tout en évitant également des pondérations résultant de la redondance de certains groupes d'indicateurs. L'analyse fine, là encore, de la représentation des indicateurs traités par les principaux facteurs a permis de préciser l'interprétation de la structure d'équipements ainsi dégagée, tout en contrôlant celle-ci par la comparaison systématique avec les analyses sectorielles, qui a montré qu'on retrouvait bien, dans la synthèse, les principaux résultats de celles-ci (Préteceille, 1979b). 
Notre intérêt particulier pour la caractérisation des espaces où s'organise la consommation et le rôle clé de ces espaces dans le rapport entre structure sociale et structure d'équipement nous a conduits, à partir de cette analyse en composantes principales, à l'élaboration d'une typologie ${ }^{6}$.

À partir de leurs coordonnées sur les quatre premiers facteurs (caractérisation plus synthétique de la structure d'équipements de chaque unité spatiale), les communes et quartiers ont été regroupés en dix-sept types. Nombre arbitraire, qui est un compromis entre la recherche de description synthétique et le désir de garder une certaine prise en compte de la diversité des distributions. On a analysé chacune de ces configurations spécifiques d'équipements à partir de la valeur moyenne des indicateurs pour chaque type. Puis la composition sociale et démographique moyenne des communes ainsi regroupées en comparant les données des recensements de 1968 et 1975 pour faire apparaitre les transformations de la population. Enfin, on a comparé ces types à une autre typologie établie par ailleurs uniquement en fonction de la structure sociale afin de mesurer leur homogénéité sociologique.

\section{Résultats et interprétations}

L'un des écueils de l'usage des méthodes d'analyse multidimensionnelle tient à la disproportion criante entre la puissance et la sophistication des méthodes de calcul et la pauvreté des moyens d'interprétation des résultats, où le bon sens et le pifomètre prennent le relais et glissent trop souvent vers des interprétations causales injustifiées, pauvres (les déterminations sociales sont des mécanismes complexes et contradictoires qui ne peuvent être réduits aux rapports entre un petit nombre de variables, fussent-elles synthétiques) voire abusives (les « interprétations » sont nécessairement limitées aux variables descriptives introduites : pourquoi celles-là et pas d'autres en plus ou à leur place...). En fait, l'explicitation des résultats d'un processus de traitement statistique est, en soi, un processus d'élaboration qu'on ne saurait réduire au seul « mapping », projection graphique des variables étudiées et des variables « explicatives » (caractéristiques des « individus » décrits) sur le plan des deux premiers facteurs de l'analyse multidimensionnelle - graphique qui fait florès actuellement, jusque dans les hebdos qui se veulent « in », mais qui caricature les résultats.

On donnera seulement ici deux exemples qui illustrent cette nécessité d'un investissement important dans l'élaboration des résultats.

Le premier est celui de l'interprétation des facteurs, c'est-à-dire de l'élaboration de leur signification comme synthèse de certains sous-ensembles des variables de départ, première étape classique. Elle nous a permis de montrer le rôle majeur, et très corrélé, du système des transports, de l'accessibilité des grands équipements publics (universités, hôpitaux, centres de formation professionnelle des adultes...), des services postaux et de télécommunication ainsi que de nombreux services privés organisant la consommation marchande premier facteur qui hiérarchise radioconcentriquement les unités spatiales, des quartiers centraux de Paris aux communes de la banlieue éloignée. Puis l'importance de la proximité des équipements publics « de voisinage » — second facteur — qui favorise la proche banlieue, opposée à Paris (bien équipé mais avec une structure différente) et à la banlieue éloignée. Mais le troisième facteur, qui représente surtout l'enseignement secondaire privé, les autres équipements de voisinage privés (sports, etc.) et la variété des options dans l'enseignement secondaire public, fait nettement éclater en deux groupes distincts cette unité de la proche banlieue.
6 Ces travaux informatiques ont été réalisés au centre de calcul du CNRS (CIRCE) à Orsay avec le concours de Jean-Pierre Fénelon, économiste au CNRS, et de PierreOlivier Flavigny, informaticien au CNRS. L'analyse en composantes principales a utilisé le programme IAG 01 de Iagolnitzer. La typologie, pour laquelle nous avons utilisé le programme PARTES de Morineau, appartient à la famille des « centres mobiles » (du type «nuées dynamiques », voir Lebart et al, 1977). 
Le second exemple est celui du croisement avec les variables descriptives de la structure de la population. La projection usuelle des CSP regroupées sur le plan des deux premiers facteurs fait apparaître un résultat fort important : la situation relativement privilégiée des catégories « supérieures » (cadres supérieurs et professions libérales), relativement défavorisée des ouvriers à l'autre extrémité, et intermédiaire des cadres moyens et des employés. Mais l'étude de la distribution des catégories socio-professionnelles plus fines dans les dix-sept types montre d'intéressantes perturbations de ces grandes tendances : par exemple, le fort pourcentage d'ouvriers dans un type bien équipé de communes de la proche banlieue, où l'on observe aussi un très faible pourcentage de cadres supérieurs et professions libérales. Il montre aussi l'éclatement de certaines grandes catégories comme les cadres moyens : les instituteurs et personnels des services médicaux et sociaux étant distribués dans les types comme les catégories supérieures « intellectuelles » liées à la reproduction sociale (professeurs, professions libérales), les techniciens étant au contraire dans une situation d'accès aux équipements très semblable à celle des ouvriers, particulièrement des contremaîtres. De plus, les tendances ségrégatives relevées ne sauraient être caricaturées en oppositions absolues : plus de $5 \%$ de la population des ménages dont le chef est ouvrier ou technicien habitent dans les sept types les mieux équipés, et dans le type le plus « bourgeois » de proche banlieue, ils représentent le tiers de la population totale...

À partir de ces analyses fines, qui permettent donc une caractérisation précise des différents espaces d'équipements et des structures sociales correspondantes ${ }^{7}$, on est ensuite conduit à l'élaboration d'un ensemble d'hypothèses spécifiques quant aux conséquences de chaque type d'espace sur l'organisation des pratiques de consommation et quant aux processus sociaux (économiques, politiques, etc.) qui ont produit ces structures d'équipements et à cette distribution socio-spatiale.

$\mathrm{Si}$, en effet, cette étude statistique apporte un éclairage nécessaire et original à la caractérisation des conditions de consommation et fournit une base solide pour l'analyse ultérieure de ces pratiques elles-mêmes, elle ne produit pas directement d'informations sur les modalités de circulation, de distribution effective et d'appropriation réelle des équipements. L'étude de leur usage ou non-usage et de leurs effets divers suppose, par exemple, qu'on prenne en compte non seulement l'accessibilité physique, mais aussi ce qu'on pourrait appeler la « distance sociale » (Pinçon-Charlot et Rendu, 1982) résultant des modalités spécifiques d'usage de l'espace et d'organisation des pratiques. Celles-ci varient selon les catégories sociales, en fonction de leurs conditions matérielles d'existence (revenu, temps, qualification, lieu et condition de travail) comme en fonction des rapports idéologiques, politiques, symboliques qu'elles entretiennent les unes avec les autres. L'étude de ces pratiques suppose donc, au-delà des hypothèses résultant de la confrontation de nos résultats aux recherches déjà effectuées dans ce domaine, des démarches nouvelles impliquant, par exemple, tout un processus d'enquêtes et d'observations locales (Pinçon, 1979b).

Quant à l'analyse des « causes » de la distribution socio-spatiale des équipements, elle implique elle aussi, au-delà des croisements statistiques avec les caractéristiques politiques et financières des municipalités (Rendu, 1979), des démarches spécifiques d'étude des rapports complexes entre État central, collectivités locales, entreprises, promoteurs, etc. qui ne sauraient être réduites aux règles institutionnelles ou à quelques variables, vu la dispersion des pratiques de ces agents (Préteceille, 1981).

Ainsi, le travail de production d'hypothèses nouvelles, à partir de la diversité et de la complexité des résultats obtenus nous oblige-t-il à transformer, sur de nombreux points, les positions théoriques de départ. Il oblige aussi, en mesurant les limites du compromis sur les données, à penser d'autres dé-
$7 \quad$ On trouvera une présentation plus détaillée des structures d'équipement et des structures sociales et démographiques de chacun des dix-sept types dans le rapport précédemment cité, ainsi que dans Pinçon-Charlot et Rendu (1981) et aussi Pinçon-Charlot et al. (1986) qui présente, outre la synthèse des résultats empiriques, l'ensemble des hypothèses interprétatives proposées. 
marches de recherches complémentaires avec d'autres méthodes : s'appuyer sur les statistiques pour leur propre dépassement, dans l'analyse des pratiques de consommation ou dans celle des processus politiques. 


\section{Bibliographie}

Briand Jean Pierre, ChAPoulie Jean Michel et PÉREtz Henri, 1979, « Les statistiques scolaires comme représentation et comme activité », Revue française de sociologie, vol. 20, n 4, p. 669-702.

Chantrein Michel, Pinçon Monique, Préteceille Edmond et Rendu Paul, 1977-1977, Indicateurs d'équipements collectifs en région parisienne, Paris, CSU, vol. 1, 1976 ; vol. 2, 1977.

DreYFus Jacques, 1975, Implication ou neutralité des méthodes statistiques appliquées aux sciences sociales, Paris, CREDOC.

LEbart Ludovic, Morineau Alain et TABARD Nicole, 1977, Techniques de la description statistique, Paris, Dunod.

PInÇON Monique, 1979a, Espace social et espace culturel. Analyse de la distribution socio-spatiale des équipements culturels et éducatifs en région parisienne, Paris, CSU, 557 p.

Pinçon Monique, 1979b, Modes de vie, cadres de vie. Structures locales d'équipements et pratiques sociales : une enquête-pilote en région parisienne, Paris, CSU, 144 p.

Pinçon-Charlot Monique et Rendu Paul, 1981, Espace des équipements collectifs et ségrégation sociale, Paris, CSU, 165 p.

Pinçon-Charlot Monique et Rendu Paul, 1982, « Distance spatiale, distance sociale aux équipements collectifs en Île-de-France : des conditions de la pratique aux pratiques », Revue française de sociologie, vol. 23, $\mathrm{n}^{\circ}$ 4, p. 667-696.

Pinçon-Charlot Monique, Préteceille Edmond et Rendu Paul, 1986, Ségrégation urbaine : classes sociales et équipements collectifs en région parisienne, Paris, Anthropos.

PrÉTECEILle Edmond, 1979a, Équipements collectifs, sciences sociales et planification. Utilité et usages d'indicateurs d'équipements collectifs, Paris, CSU, $42 \mathrm{p}$.

PréteCEILle Edmond, 1979b, Équipements collectifs, classes sociales et structures urbaines, Paris, CSU, $84 \mathrm{p}$.

Préteceille Edmond, 1981, « Left using local authorities and services policy in France », International journal of urban and regional research, vol. 5, $\mathrm{n}^{\circ}$ 3, p. 411-425.

Préteceille Edmond, PinÇOn-Charlot Monique et Rendu Paul, 1975, Équipements collectifs structures urbaines et consommation sociale, Paris, CSU, 163 p.

RENDU Paul, 1979, Équipements collectifs, gestion financière et couleur politique des grandes communes de la banlieue parisienne, Paris, CSU, $42 \mathrm{p}$. 